\title{
Electron dense staining affinities of mouse oxytalan and elastic fibers
}

\author{
T. A. Simmons And J. K. Avery \\ Laboratory of Oral Histology, Department of Oral Biology, The University of Michigan, \\ Ann Arbor, MI., U.S.A.
}

\begin{abstract}
The staining affinity of electron dense stains to mouse palatal elastic fibers and periodontal oxytalan fibers was assessed and compared. Tissues were stained routinely with uranyl acetate and lead citrate or with elastic tissue stains including: (1) silver tetraphenylporphine sulfonate, (2) tannic acid technique, and (3) a high iron diamine sequence. Staining results were assessed with an electron microscope. Palatal elastic fibers demonstrated staining affinity to all elastic stain techniques. Oxytalan fibers did not demonstrate affinity to elastic fiber stains used. Based on electron dense stains, elastic and oxytalan fibers were dissimilar, in contrast to results reported utilizing light microscopic stains.
\end{abstract}

Accepted for publication 9 November 1979

The oxytalan fiber is a connective tissue fiber of the periodontal ligament first described by Fullmer \& Lillie (1958). The oxytalan fiber has often been confused with elastic fibers due to (1) the demonstration of these fibers with elastic tissue stains, (2) the similar morphology observed with the light microscope, and (3) the close microfibrillar resemblance observed in ultrastructure. Oxytalan fibers are stained with Gomori's aldehyde fuchsin after preoxidation with peracetic acid or potassium monopersulfate. Since elastic fibers are also stained following this method, it is necessary to stain alternate sections without preoxidation to determine the presence of elastic fibers.

In ultrastructure both the oxytalan and elastic fibers display a composition of parallel arranged fibrils approximately

This work was supported by a BRS Grant \#S501RRO5321-10.

0300-9777/80/040183-06\$02.50/0
$150 \AA$ in diameter (Carmichael \& Fullmer 1966). The only distinguishing characteristic of elastic fibers using the standard E.M. stains uranyl acetate and lead citrate is the appearance of an amorphous (elastin-rich) zone of the elastic fiber (Pease \& Molinaris 1960). The appearance of this zone is dependent on the maturity of the fiber and the plane of sectioning.

Pre-elastic or oxytalan fibers do not stain with elastic tissue stains unless a preoxidation step is employed. In addition, the stainable component of elastic fibers is removed by beta-glucuronidase following oxidation, as it is with oxytalan fibers (Fullmer 1960a). All investigations to characterize oxytalan fibers have utilized elastic tissue stains following preoxidation of the tissue sections resulting in their subsequent visualization by light microscopy.

Recently, new electron dense stains c 1980 Munksgaard, Copenhagen 
have been introduced in order to facilitate the identification of elastic fibers. Most of these stains selectively stain the elastin or amorphous zone of the elastic fiber. However, one stain developed by Spicer et al. (1975) selectively appears to impart density to the microfibrillar component of elastic fibers. It is the purpose of this study to determine the affinity of these electron dense stains to the fine fibers oriented in the long axis of the mouse periodontal ligament and compare them with mouse palatal elastic fibers similarly stained.

\section{Material and Methods}

Two biopsies were taken from ten 60-dayold Swiss-Webster mice. The biopsies, comprised of tooth, periodontal ligament, and alveolar bone, were taken by carefully dissecting the mandible, removing all soft tissue attachments, and splitting the first molar and the associated bone mesial and distal to the molar, thus obtaining two biopsies from each side of the mandible. A full thickness palatal biopsy was taken from the hard palate. The tissues were fixed by three procedures as follows:

\section{Routine Fixation}

1. The tissue was fixed $1 \mathrm{~h}$ in a $2.6 \%$ glutaraldehyde in S-collidine buffer at pH 7.2.

2. Rinsed in buffer, postfixed $2 \mathrm{~h}$ in $1.33 \% \mathrm{OsO}_{4}$.

3. Rinsed and dehydrated with 2,2,dimethoxypropane.

4. Infiltrated and embedded in Epon 812.

\section{Tannic Acid-Glutaraldehyde Staining} Fixative (Cotta-Pereira et al. 1976)

1. The tissue was fixed $1 \mathrm{~h}$ in $0.25 \%$ tannic acid in $3 \%$ glutaraldehyde in Millonig's buffer.
2. Rinsed in buffer, postfixed in $1.33 \%$ $\mathrm{OsO}_{4}$ in Millonig's buffer.

3. Rinsed and dehydrated with 2,2, dimethoxypropane.

4. Infiltrated and embedded in Epon 812.

High Iron Diamine Staining Sequence for Microfibrillar Component (Spicer et al. 1975)

1. The tissue was fixed $1 \mathrm{~h}$ in a $2.6 \%$ glutaraldehyde in S-collidine buffer at $\mathrm{pH}$ 7.2.

2. Rinsed in buffer, immersed in a solution containing $0.25 \% \mathrm{KMnO}_{4}$ and $0.25 \%$ $\mathrm{H}_{2} \mathrm{SO}_{4}$.

3. Rinsed with distilled water, stained overnight in a solution of $120 \mathrm{mg}$ of $\mathrm{N}, \mathrm{N}$,-dimethyl-m-phenylenediamine dis. solved simultaneously with $20 \mathrm{mg}$ of $\mathrm{N}, \mathrm{N}$,-dimethyl-p-phenylenediamine in $50 \mathrm{ml}$ of distilled water. When dis. solved, $1.4 \mathrm{ml}$ of $10 \% \mathrm{FeCl}_{3}$ was added.

4. The tissue was rinsed in distilled water, postfixed in $1.33 \% \mathrm{OsO}_{4}$ for $2 \mathrm{~h}$.

5. Rinsed and dehydrated with 2,2, dimethoxypropane.

6. Infiltrated and embedded in Epon 812

Routine glutaraldehyde fixed tissues were then thin sectioned and examined without staining. The routinely fixed tissues were also stained with two methods: (1) with uranyl acetate and lead citrate which enhances overall staining, and (2) with a $10 \%$ solution of silver tetraphenylporphin sulfonate at $60^{\circ} \mathrm{C}$ for $30 \mathrm{~min}$ on formvar coated grids to illustrate the amorphous component (Albert \& Fleischer 1970). Tissues processed with the high iron diamine method were sectioned and examined in the electron microscope without further staining. All determinations of staining affinities were made using a Zeiss EM 9S 2 electron microscope. 


\section{Results}

The tissues which were treated only with glutaraldehyde and osmium tetroxide showed no contrast nor staining density. Determination of staining affinities was made while observing in the electron microscope, not from printed photographs. Elastic fibers and oxytalan fibers appeared very light without contrast. Routine fixation of mouse palatal tissue, which contains many elastic fibers, followed by uranyl acetate and lead citrate, stained the microfibrillar portion of the fibrotubular elastic fibers (Fig. 1). The amorphous zone of mature elastic fibers did not stain. Mouse periodontal ligament oxytalan fibers stained with uranyl acetate and lead citrate stained both the collagen fibers and oxytalan fibers (Fig. 2).

Tannic acid fixation utilizing $0.25 \%$ tannic acid solution showed positive staining of the amorphous region of the elastic fiber (Fig. 3). Tannic acid did increase the density of elastic fiber microfibrils slightly; however, oxytalan fibers (Fig. 4) were not easily recognized on sections when stained with tannic acid alone.

The amorphous zone of elastic fibers was stained and contained many darkly staining particles resembling a stain precipitate (Fig. 5). Oxytalan fibers did not stain with darkly staining particles (Fig. 6).

The high iron diamine method, as described by Spicer et al. (1975), stained a peripheral zone which corresponds in distribution with the microfibrillar component surrounding the large elastic fibers of the deep dermis. In the mouse palatal tissue both the microfibrillar and the amorphous component of the elastic fibers were stained by this method (Fig. 7). Oxytalan fibers were not stained by this method and exhibited slight contrast. Tissues which were stained by this method were examined under higher magnifications in order to help identify oxytalan fibers (Fig. 8). Sections were then stained with uranyl acetate and lead citrate in order to check the tissue sections for identification of oxytalan fibers.

\section{Discussion}

The oxytalan fiber has often been described electron microscopically as consisting of microfibrils similar in appearance to the microfibrillar component of the elastic fiber (Carmichael \& Fullmer 1966, Griffin \& Harris 1967, Greenlee et al. 1966, Ross \& Bornstein 1969, Williams 1970). Oxytalan fibers may also contain an amorphous ground substance. Following preoxidation with peracetic acid or potassium monopersulfate and treatment with beta-glucuronidase, the aldehyde fuchsin stainability is lost and a visible residue remains (Fullmer 1960b).

In the present study electron dense stains were used in the study of both the microfibrillar and amorphous components of the tissue. The use of various electron dense stains may offer greater insight into the nature of these fibers once the staining mechanism is better understood.

Oxytalan fibers did not stain with any of the special stains used. This may be viewed as evidence that oxytalan fibers have both an amorphous and microfibrillar component different from elastic fibers. A possible explanation for the difference may be a masking of the reactive site on the oxytalan fiber by another substance or site. It is possible that preoxidation of the tissue, followed by electron dense staining techniques, may demonstrate staining affinity. The high iron diamine technique developed by Spicer et al. (1975) requires a $5 \mathrm{~min}$ bath of $0.25 \%$ potassium permanganate following glutaraldehyde 

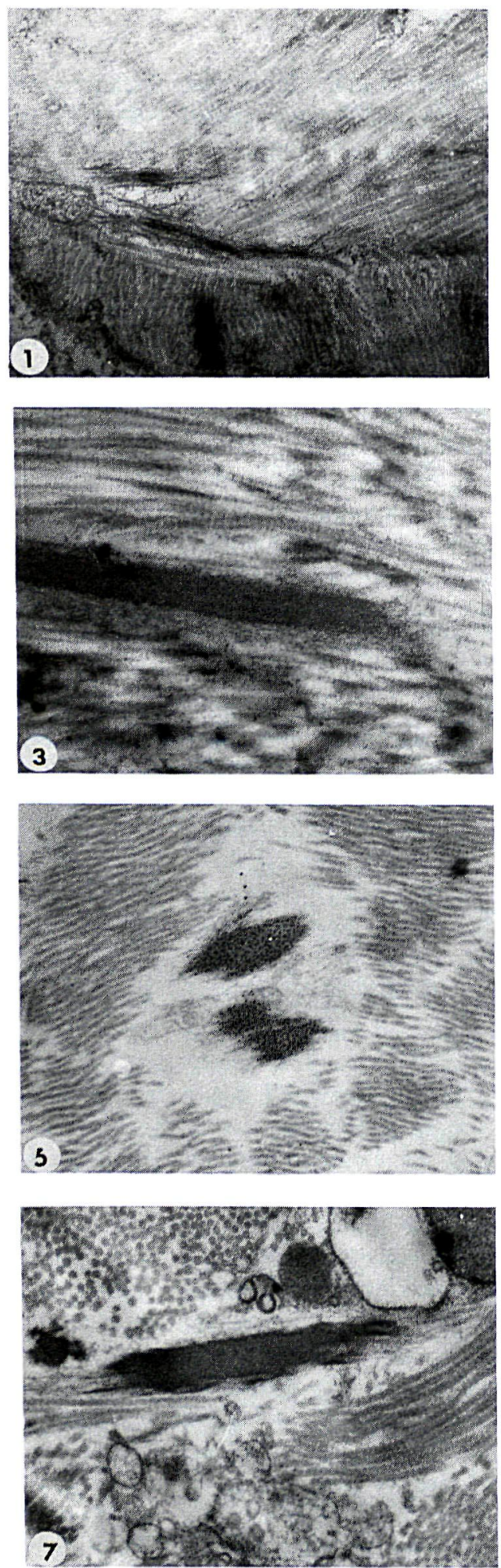
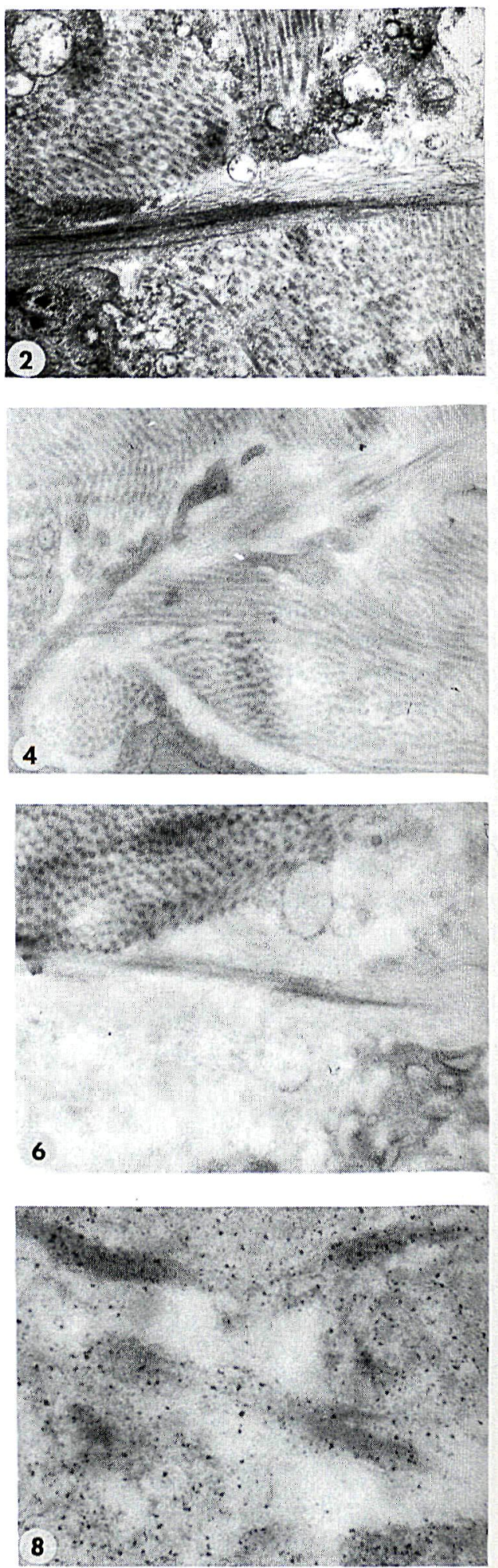


\begin{tabular}{|l|c|c|c|c|}
\hline & \multicolumn{2}{|c|}{ ELASTIC FIBER } & \multicolumn{2}{|c|}{ OXYTALAN FIBER } \\
\hline $\begin{array}{l}\text { Micro- } \\
\text { fibrils }\end{array}$ & $\begin{array}{c}\text { Amorphous } \\
\text { Zone }\end{array}$ & $\begin{array}{c}\text { Micro- } \\
\text { fibrils }\end{array}$ & $\begin{array}{c}\text { Amorphous } \\
\text { Zone }\end{array}$ \\
\hline $\begin{array}{l}\text { Routine fixation } \\
\text { noutine fixation uranyl }\end{array}$ & + & - & - & - \\
\hline $\begin{array}{l}\text { Tannic acid } \\
\text { fixation and staining }\end{array}$ & + & + & + & - \\
\hline $\begin{array}{l}\text { Routine fixation } \\
\text { Ag-tetraphenylporphine } \\
\text { sulfonate staining }\end{array}$ & - & + & - & - \\
\hline $\begin{array}{l}\text { Permanganate high iron } \\
\text { diamine fixation and } \\
\text { staining }\end{array}$ & + & + & - & - \\
\hline
\end{tabular}

Fig. 9. Summary of electron dense stains tested. + indicates positive staining.

fixation prior to the iron treatment. iron diamine method has been described as Mander et al. (1967) showed that a $0.25 \%$ having an affinity primarily for the potassium permanganate solution microfibrillar component of elastic fibers. effectively oxidized mouse periodontal In this study both the amorphous and ligament prior to aldehyde fuchsin staining microfibrillar component of mouse elastic to demonstrate oxytalan fibers. The high fibers showed electron density to the high

Fig. I. Mouse palatal elastic fiber. Routine fixation, uranyl acetate and lead citrate staining. Mag. $\times 16,700$.

Fig. 2. Mouse periodontal ligament oxytalan fiber. Routine fixation, uranyl acetate and lead citrate staining. Mag. $\times 16,700$.

Fig. 3. Mouse palatal elastic fiber showing staining of amorphous zone. Tannic acid fixation, no further staining. Mag. $\times 16,700$.

Fig. 4. Mouse periodontal oxytalan fiber. Tannic acid fixation, no further staining. Mag. $\times 16,700$.

Fig. 5. Staining and dark precipitate on mouse palatal elastic fiber. Routine fixation and silver tetraphenylporphine sulfonate staining. Mag. $\times 16,700$.

Fig. 6. Mouse periodontal ligament oxytalan fiber. Routine fixation and silver tetraphenylporphine sulfonate staining. Mag. $\times 16,700$.

Fig. 7. Staining of amorphous and microfibrillar components of mouse palatal elastic fiber. High iron diamine fixation, no further staining. Mag. $\times 16,700$.

Fig. 8. High magnification of mouse periodontal oxytalan fiber, no staining of microfibrils. High iron diamine fixation, no further staining. Mag. $\times 49,300$. 
iron diamine stain. The oxytalan fibers did not react with the high iron diamine stain.

Presently the only electron dense stain which does provide adequate contrast to the oxytalan fiber is uranyl acetate, and much greater contrast develops when it is combined with lead citrate. The electron dense elastic tissue stains tested react dissimilarly when used on elastic fibers and oxytalan fibers. Oxytalan fibers exhibit no staining of the ground substance with these stains. A summary of affinities of stains tested is presented in Fig. 9.

\section{References}

Albert, E. N. \& Fleischer, E. (1970) A new electron dense stain for elastic tissue. Journal of Histochemistry and Cytochemistry 18, 697-708.

Carmichael, G. G. \& Fullmer, H. M. (1966) The fine structure of the oxytalan fiber. Journal of Cell Biology 28, 33-36.

Cotta-Pereira, G., Rodrigo, F. G. \& David-Ferreira, J. F. (1976) The use of tannic acidglutaraldehyde in the study of elastic and elastic related fibers. Stain Technology 51, 7-11.

Fullmer, H. M. (1960a) Observations on the development of oxytalan fibers in radicular cysts and dental granulomas. A.M.A. Archives of Pathology $70,59-67$.

Fullmer, H. M. (1960b) Effect of peracetic acid on the enzymatic digestion of mucopolysaccharides: reversal of the PAS staining reaction of mucin. Journal of Histochemistry and Cytochemistry 8, $113-121$
Fullmer, H. M. \& Lillie, R. D. (1958) The oxytalan fiber: a previously undescribed connective tissue fiber. Journal of Histochemistry and Cytochemistry 6, 426-430.

Greenlee, T. K., Ross, R. \& Hartman, J. C. (1966) The fine structure of elastic fibers. Journal of Cell Biology 30, 59-71.

Griffin, C. J. \& Harris, R. (1967) The fine structure of the developing human periodontium. Archives of Oral Biology 12, 971-982.

Mander, S. T. K., Mander, L. N. \& Carmichael, G. G. (1967) The staining mechanism of aldehydefuchsin with reference to the oxytalan fiber in the mouse. Journal of Histochemistry and Cytochemistry 10(7), 480-485.

Pease, D. \& Molinaris, S. (1960) Electron microscopy of muscular arteries; pial vessels of the cat and monkey. Journal of Ultrastructure Research 3, 447-468.

Ross, R. \& Bornstein, P. (1969) The elastic fiber. I. The separation and partial characterization of its macromolecular components. Journal of Cell Biology 40, 366-381.

Spicer, S. S., Brissie, R. M. \& Thompson, N. T. (1975) Variability of dermal elastin visualized ultrastructurally with iron hematoxylin. American Journal of Pathology 79, 481-496.

Williams, G. (1970) The late phases of wound healing: histological and ultrastructural studies of collagen and elastic-tissue formation. Journal of Pathology 102, 61-68.

Address:

J. K. Avery

Laboratory of Oral Histology

Department of Oral Biology

The University of Michigan

Ann Arbor, Michigan 48109

U.S.A. 
This document is a scanned copy of a printed document. No warranty is given about the accuracy of the copy. Users should refer to the original published version of the material. 